\title{
Simulations of Laser Imprint for Nova Experiments and for Ignition Capsules
}

\author{
S. V. Weber \\ S. G. Glendinning \\ D. H. Kalantar \\ M. H. Key \\ B. A. Remington \\ J. E. Rothenberg \\ E. Wolfrum \\ C. P. Verdon \\ J. P. Knauer
}

This paper was prepared for submittal to the 38th Annual Meeting, American Physical Society

Division of Plasma Physics

Denver, Colorado

November 11-15, 1996

November 8, 1996

This is a preprint of a paper intended for publication in a journal or proceedings. Since changes may be made before publication, this preprint is made available with the understanding that it will not be cited or reproduced without the permission of the author. 


\section{DISCLAIMER}

This document was prepared as an account of work sponsored by an agency of the United States Government. Neither the United States Government nor the University of California nor any of their employees, makes any warranty, express or implied, or assumes any legal liability or responsibility for the accuracy, completeness, or usefulness of any information, apparatus, product, or process

disclosed, or represents that its use would not infringe privately owned rights. Reference herein to any specific commercial product, process, or service by trade name, trademark, manufacturer, or otherwise, does not necessarily constitute or imply its endorsement, recommendation, or favoring by the United States Government or the University of California. The views and opinions of authors expressed herein do not necessarily state or reflect those of the United States Government or the University of California, and shall not be used for advertising or product endorsement purposes. 


\title{
Simulations of laser imprint for Nova experiments and for ignition capsules
}

\author{
S.V. Weber, S.G. Glendinning, D.H. Kalantar, M.H. Key, B.A. Remington, J.E. \\ Rothenberg, \\ Lawrence Livermore National Laboratory, \\ Livermore, California 94550 \\ E. Wolfrum, \\ Rutherford Appleton Laboratory, Chilton, Didcot, United Kingdom \\ C.P. Verdon, and J.P. Knauer \\ Laboratory for Laser Energetics, University of Rochester, Rochester, NY 14623
}

(November 8, 1996)

\begin{abstract}
In direct drive ICF, nonuniformities in laser illumination seed ripples at the ablation front in a process called "imprint". These nonuniformities grow during the capsule implosion and, if initially large enough, can penetrate the capsule shell, impede ignition, or degrade burn. Imprint has been simulated for recent experiments performed on the Nova laser at LLNL examining a variety of beam smoothing conditions. Most used laser intensities similar to the early part of an ignition capsule pulse shape, $I \cong 10^{13} \mathrm{~W} / \mathrm{cm}^{2}$. The simulations matched most of the measurements of imprint modulation. The effect of imprint upon National Ignition Facility (NIF) direct drive ignition capsules has also been simulated. Imprint is predicted to give modulation comparable to an intrinsic surface finish of $\sim 10 \mathrm{~nm}$ RMS. Modulation growth was ex-
\end{abstract}


amined using the Haan [Phys. Rev. A 39, 5812 (1989)] model, with linear growth factors as a function of spherical harmonic mode number obtained from an analytic dispersion relation. Ablation front amplitudes are predicted to become substantially nonlinear, so that saturation corrections are large. Direct numerical simulations of two-dimensional multimode growth were also performed. The capsule shell is predicted to remain intact, which gives a basis for believing that ignition can be achieved.

52.50.Jm, 52.58.Ns, 52.35.Py, 52.35.-g

Typeset using REVTEX 


\section{INTRODUCTION}

In direct drive inertial confinement fusion, spatial nonuniformities in the laser intensity cause modulations in ablation pressure, which in turn generate hydrodynamic perturbations in the target being driven. This process is called "imprint". The favored method of minimizing imprint is use of laser beam smoothing schemes ${ }^{1-3}$ which introduce spatial and temporal incoherence. These create a speckle intensity pattern at any instant of time which is strongly modulated, but the pattern varies in time in a nearly random manner so that the modulation smooths out.

Thermal transport between where the laser energy deposits and the ablation front gives additional smoothing. This thermal smoothing has been called the "cloudy day" effect." Modulation of ablation pressure is predicted to be reduced from the modulation in laser intensity as

$$
\delta P / P=e^{-k \Delta r}
$$

where $P$ is the ablation pressure, $k$ is the wavenumber of the transverse intensity modulation, and $\Delta r$ is the separation between where the laser energy is deposited and the ablation front. Note that ICF ignition benefits from a short laser wavelength and a low intensity $\left(<10^{14} \mathrm{~W} / \mathrm{cm}^{2}\right)$ foot for the temporal profile of the drive. For such conditions, most of the light is absorbed at densities significantly below the critical density, so Eqn. 1 should be convolved over the range of deposition locations. This process is somewhat more complex to calculate accurately because it takes time for beam smoothing schemes to operate, and the structure both of the plasma corona where the laser deposition and thermal smoothing occur, and the hydrodynamic response of the target being driven, change in time.

Imprint has been examined in experiments at several laser facilities ${ }^{5-13}$ and in simulations. ${ }^{14}$ We have modeled Nova experiments to test our understanding of the physics of imprint and to verify our ability to predict the results. We will show good agreement of simulations with experiments at Nova measuring imprint upon $\mathrm{CH}_{2}$ foils at intensities of 
$10^{13}-10^{14} \mathrm{~W} / \mathrm{cm}^{2}$ driven with $0.35 \mu \mathrm{m}$ or $0.53 \mu \mathrm{m}$ light. Experiments done at Nova or at the Vulcan laser at Rutherford Laboratory using XUV laser probes are more complicated to understand. Although the XUV probe gives high spatial resolution and superior sensitivity, the opacity of the sample to the probe wavelength depends upon the sample density. Furthermore, the targets for these experiments were $\mathrm{Si}$ or $\mathrm{Al}$ in order to get adequate signal though the foils at the probe wavelength. These materials emit x-rays from the corona which cause preheat of the foils. It is more difficult to model all of these effects accurately.

We have applied the same simulation methods to assess effects of imprint upon direct drive capsule designs intended to achieve ignition at the National Ignition Facility (NIF). We find that the modulation due to imprint should be comparable to an achievable surface finish of $\sim 10 \mathrm{~nm}$ in spherical harmonic modes $l>10$. We shall discuss Rayleigh-Taylor (RT) growth of these ablation front modulations during the implosion. We find that although RT linear growth factors reach several thousand, when saturation is considered the capsule shell is predicted to remain intact and the capsule to ignite.

We shall discuss our methods in Section II. In Section III we will discuss simulations of Nova experiment using $\mathrm{CH}_{2}$ foils, and Section IV we shall discuss Nova and Vulcan experiments using XUV laser probes. In section V, analysis of the NIF capsule design will be presented. Section VI gives conclusions.

\section{NUMERICAL METHOD}

Our modeling has been done using the computer code LASNEX. ${ }^{15}$ Simulations are twodimensional with reflecting boundary conditions in the transverse direction, and include multigroup radiation diffusion, electron conduction by flux-limited diffusion with a flux limiter of $f_{e}=0.1$, and ray trace laser deposition. The laser ray trace does not give interference, so the speckle intensity modulation is introduced by adjusting the powers of the rays according to their aiming point at the surface of the target. Rays were directed at the target at a range of angles of incidence corresponding to the focal ratio of the illumination. The ray 
set was modified every cycle in a Monte Carlo fashion to minimize statistical fluctuations in laser intensity due to the finite number of rays.

After some time, the growth history of a perturbation resulting from laser speckle is found to parallel that of a perturbation originating at the surface of the target. ${ }^{6}$ The growth usually becomes parallel a short time after shock breakout of the foil or shell, during the bulk acceleration. Imprint is largely complete by that time for the conditions which we have considered, and perturbations from any source relax into eigenmodes of the RT instability. The growth history may not be parallel during shock transit even if imprint is over because the longitudinal spatial structure of the perturbation may depend on how it is created during that phase of the evolution. We have describe the amplitude of imprinted modulation by relating it to the amplitude of a surface perturbation which produces a similar effect during the phase of parallel RT evolution. This is called the equivalent surface finish,

$$
\varepsilon(k)=\frac{\eta_{\text {imprint }}(k, t)}{\eta_{\text {surface }}(k, t)} \eta_{\text {surface }}(k, t=0)
$$

Here, $\eta$ is the amplitude of modulation, assessed in any consistent manner, for modulation due to imprint or surface perturbations, at modulation wave number, $k$, and time, $t$, during the parallel phase of evolution.

Imprinting ceases for either of two reasons. The deposition standoff may become so large that the ablation pressure modulation becomes small. Alternatively, hydrodynamic growth such as by RT, can amplify the effect of imprint which occurred earlier in time so that it overwhelms the effect of additional imprint which occurs later. We shall show this effective termination of imprint later in this paper.

Imprinted modulation is proportional to the amplitude of the laser intensity modulation which caused it if the hydrodynamic perturbations are linear in amplitude. Therefore, we define an imprint efficiency as the equivalent surface finish divided by the amplitude of the laser intensity modulation,

$$
E(k)=\varepsilon(k) / \frac{\delta I(k)}{I}
$$


where $\delta I(k) / I$ is the fractional modulation of laser intensity at wavenumber, $k$. The imprint efficiency may be calculated at some $\delta I(k) / I \ll 1$, so that the response remains linear. The result is multiplied by the intensity modulation for a case of interest to determine the modulation amplitude.

\section{SIMULATIONS OF NOVA $\mathrm{CH}_{2}$ EXPERIMENTS}

Experiments have been carried out on the Nova laser in which $\mathrm{CH}_{2}$ foils were accelerated by one smoothed beam in the two beam chamber and modulation was diagnosed by face-on $x$-ray radiography using gated pinhole imaging. ${ }^{13,6,16,17}$ One recent experiment ${ }^{13}$ compared imprint of static speckle, using a random phase plate ${ }^{1}$ (RPP) but no laser bandwidth, between $0.35 \mu \mathrm{m}$ and $0.53 \mu \mathrm{m}$ illumination at $\sim 10^{13} \mathrm{~W} / \mathrm{cm}^{2}$. The $\mathrm{f} /$ number of the illumination was adjusted, $\mathrm{f} / 16.5$ for $0.35 \mu \mathrm{m}$ and $\mathrm{f} / 11$ for $0.53 \mu \mathrm{m}$ to obtain the same size speckles for both cases. A surface perturbation of $50 \mu \mathrm{m}$ wavelength and $2 \mu \mathrm{m}$ amplitude was imposed upon the foils to allow extraction of the equivalent surface finish. This does not quite correspond to Eqn. 2 because modulation for the same surface finish wavelength was used to divide imprint at different wavelengths. The drive laser intensity pattern was measured and used to deduce the imprint efficiency at the predicted shock breakout time, 2 ns for $30 \mu \mathrm{m}$ thick foils.

Figure 1 shows the modal amplitude spectrum of imprint efficiency for the two illumination wavelengths. Note that the efficiency is higher for $0.35 \mu \mathrm{m}$. The smaller standoff distance gives less thermal smoothing than for $0.53 \mu \mathrm{m}$ illumination. We modeled this experiment by performing 2-D single mode imprint simulations and post-processing to obtain the optical depth modulation (including instrumental resolution). Then we divided by the simulated evolution of the surface perturbation to get the equivalent surface finish, then by the applied $\delta I / I$ to obtain the imprint efficiency. The results are shown as symbols in

Figure 1, connected by lines to guide the eye. Agreement is generally quite good. There is greater disagreement at lowest mode numbers. However, experimental uncertainly is largest 
for those wavelengths as data which is not actually periodic has been Fourier transformed in a finite size box. Also, only a small number of wavelengths for such modes are present within the box, so there is a large sampling error.

We have examined the imprint time scale for the conditions of this experiment by performing simulations in which the imprint modulation was switched off at some time, and the simulation was continued with spatially uniform illumination. Column density perturbation amplitude vs. time for the case of $100 \mu \mathrm{m}$ modulation wavelength and $0.35 \mu \mathrm{m}$ illumination are shown in Figure 2. Note that little additional perturbation developed after 1 ns when the modulation was applied at all times, compared to the case where the drive was uniform after $1 \mathrm{~ns}$. That shows that imprint is nearly complete by $1 \mathrm{~ns}$ for this case. The imprinted perturbation amplitude is smaller for cases in which when the modulation was shut off earlier. Also note that the perturbation amplitude continued to grow even after the laser modulation was shut off, due to hydrodynamic evolution alone. Therefore, the modulation amplitude of the different cases should be compared at the same time, such as at the 2 ns shock breakout time, marked with x's in the figure.

Figure 3 shows imprint efficiency vs. imprinting time (i.e., time of modulation shut off) for modulation wavelengths of $30 \mu \mathrm{m}$ and $100 \mu \mathrm{m}$, and for the two laser wavelengths. We see that imprint continues longer for longer modulation wavelength or for shorter laser wavelength. This is explained qualitatively by the cloudy day effect, Eqn. 1. Longer modulation wavelengths are smoothed less, and shorter laser wavelength gives a smaller standoff distance. The standoff distance at $0.5 \mathrm{~ns}$ (average over deposition rate) is $32 \mu \mathrm{m}$ for 0.35 $\mu \mathrm{m}$ and $42 \mu \mathrm{m}$ for $0.53 \mu \mathrm{m}$, while the critical density standoffs are $1.3 \mu \mathrm{m}$ and $3.5 \mu \mathrm{m}$, respectively.

Glendinning et al. ${ }^{6}$ have also compared imprint with $1-\mathrm{D} \mathrm{SSD}^{2}$ and varying laser bandwidth, for $0.53 \mu \mathrm{m}$ illumination at a peak intensity of $\sim 10^{14} \mathrm{~W} / \mathrm{cm}^{2}$ in a ramped pulse. This case was simulated in two dimensions (2-D) using an accurate model for the multimode time-varying speckle pattern. Of course, the actual structure is 3 - $\mathrm{D}$ in this case. Figure 4 shows the RMS equivalent surface finish vs. laser bandwidth measured from the data and 
deduced from the simulations. Agreement is good. The modal spectrum from such simulations is noisy since only one mode is represented at each wavelength, and the intensity amplitude driving that mode is one sample from a random ensemble. Generally, we have achieved good agreement of simulations with the Nova experiments using $\mathrm{CH}_{2}$ foils.

\section{EXPERIMENTS USING XUV PROBING}

Kalantar and collaborators ${ }^{7},{ }^{10}$ have performed experiments at the Nova and Vulcan lasers, measuring imprinted modulation on thin foils with XUV laser probes. The Nova experiment measured imprint upon $3 \mu \mathrm{m}$ - thick Si foils with a Y probe of $15.5 \mathrm{~nm}$ wavelength, while the Vulcan experiment used $2 \mu \mathrm{m}$ - thick Al foils and a Ge probe of 19.6 $\mathrm{nm}$ wavelength. The Nova experiment used $0.35 \mu \mathrm{m}$ illumination and RPP or 1-D SSD smoothing, while the Vulcan experiments examined several smoothing options at $0.53 \mu \mathrm{m}$ illumination. The XUV probes combined with imaging using multilayer mirrors give high spatial resolution and sensitivity to small modulation amplitudes. The materials were chosen to give low absorption at the probe wavelengths, but the sample thicknesses were still small so that a detectable signal could penetrate the foils.

The experimental conditions created two complications which made modeling of these experiments more difficult. First, the opacity $\left(\mathrm{cm}^{2} / \mathrm{g}\right)$ of the samples to the probe increases with increasing sample density. The opacity is dominated by inverse bremsstrahlung absorption from a degenerate distribution of electrons from the $n=3$ shell. Tabulated opacities from the OPAL mode ${ }^{18}$ were used in the modeling. The optical depth depends upon the density structure in the sample, not just to the column density as is the case for the higher energy backlighter used in the $\mathrm{CH}_{2}$ experiments.

The other complication is that these materials emit significant X-rays from the hot coronal plasma which preheat the unablated portion of the foil. The coronal plasma is in Non-LTE conditions, which were modeling with average-atom XSN ${ }^{19}$. Data and simulations

of the RMS modulation in optical depth at shock breakout time in the Nova experiments 
are compared in Figure 5. Calculations and experiment give nearly the same result for zero laser bandwidth. However, modulation predicted for $0.33 \mathrm{THz}$ bandwidth is appreciably smaller than the measured value.

The Vulcan experiments examined illumination with static RPP speckle, 1-D SSD, induced spatial incoherence (ISI), ${ }^{3}$ and single wavelength sinusoidal illumination modulation generated by two slit interference. A reasonably complete time history was measured for each case. Experimental results are presented elsewhere in this volume ${ }^{10}$ and simulations are in progress.

\section{MODELING OF IMPRINT AND MODULATION GROWTH FOR NIF}

The National Ignition Facility is a laser capable of generating 1.8 MJ of $0.35 \mu \mathrm{m}$ light, being built by the U.S. Department of Energy for defense and ICF applications. Although the main ignition option for NIF utilizes indirect drive ${ }^{20}$, the NIF can be configured to perform direct drive implosions, and direct drive ignition appears to be achievable. We have examined a capsule design originated by one of us (CPV) to see the effects of imprint upon the implosion.

The capsule design, shown is Figure 6 , is a shell of frozen DT enclosing DT vapor, driven with 1.5 MJ of laser light. The shaped pulse designed to place the fuel on an adiabat, $\alpha=3$. The adiabat is the ratio of the pressure to the Fermi degenerate pressure. RT perturbation growth factors for the $\alpha=3$ design are below those for $\alpha=1$ designs, so that shell breakup is prevented ${ }^{21}$. Actual capsules would need a coating such as a few microns of $\mathrm{CH}$ for mechanical support, but simulations ${ }^{21}$ indicate that substitution of such a layer for the same mass of DT has little effect upon the dynamics. The capsule yield in a 1-D LASNEX simulation is 17 MJ.

The imprint efficiency was calculated for different spherical harmonic mode numbers, $l$, as discussed in Section III. The simulations were performed in planar geometry as convergence effects are small during the imprinting time. The modulation wavelength is related to the 
mode number according to the initial capsule radius, $\lambda=2 \pi r_{0} / l$. Also, these calculations did not use laser ray trace, which gave too much numerical noise. Instead, modulation of laser deposition as a function of transverse coordinate was applied to a 1-D deposition profile. The imprint efficiency vs. $l$ is shown in Figure 7.

The timescale of imprint was evaluated by shutting off the intensity modulation at different times, again as described in Section III. We looked at wavenumbers $l=30$ and $l=100$, and found timescales for generation of $80 \%$ of the total modulation of 1.4 an 4.9 ns, respectively.

An equivalent surface finish modal spectrum is obtained by multiplying the imprint efficiency by the predicted spectrum of fractional optical modulation. The optical smoothing has been analyzed in detai ${ }^{22}$ for realistic beam smoothing implementations. Here we assume a simplified smoothing in which an independent speckle pattern is generated every laser coherence time. We take the imprint timescale to be $2 \mathrm{~ns}$, independent of $l$, and a coherence time of 2 ps, from the NIF direct drive bandwidth of 0.5 THz. Overlap of 50 beamlets and two polarizations at any spot on the capsule gives an additional smoothing factor of 10 . The equivalent surface finish spectrum, shown in Figure 8, is comparable in amplitude to the surface finish from fabrication measured for Nova capsules. ${ }^{27}$ The RMS modulation from imprint is $11 \mathrm{~nm}$ and that from fabrication is $8.4 \mathrm{~nm}$, adding in RMS to a total effective surface finish of $13.9 \mathrm{~nm}$. These spectra do not include a rise in the fabrication spectrum at low mode numbers $(l<16)$ neglected in the fit shown here, or intensity modulation in low modes due to beam pointing and power imbalance.

Growth at the ablation front during the implosion may be evaluated from a dispersion relation ${ }^{23},{ }^{24}$

$$
\gamma(k)=\sqrt{\frac{k g}{1+k L}}-3 k v_{a},
$$

where $\gamma(t)$ is the growth rate, $g$ is the acceleration, $L$ is the density gradient scale length, and $v_{a}$ is the ablation velocity. Average parameters for the shell acceleration from a 1-D implosion simulation are $g=8.5 \times 10^{15} \mathrm{~cm} / \mathrm{s}^{2}, L=0.5 \mu \mathrm{m}, v_{a}=3.4 \times 10^{5} \mathrm{~cm} / \mathrm{s}$, and 
$k=0.1 \mathrm{~cm} / l$. The peak growth rate is about $2 n s^{-1}$ at $l \simeq 150$. The total linear growth factor to the time of peak inward shell velocity is $G F(k)=e^{\gamma \Delta t}$, where $\Delta t=4.66 n s$ is the acceleration time. The resulting peak linear growth factor is about 7000 .

The modal amplitudes for linear grow th were obtained by multiplying the effective surface finish spectrum by the growth factors. The ablation front perturbation spectrum at peak shell velocity is shown in Figure 9. According to the multimode saturation theory of $\operatorname{Haan}^{25}$, modes with amplitudes greater than $\nu r / l$ will be affected by saturation. We used $\nu=2$ for the saturation parameter. ${ }^{26}$ Modes near the peak of the linear growth spectrum are substantially affected by saturation. The peak in the amplitude spectrum after saturation effects are considered is shifted down to $l \simeq 60$. The total RMS amplitude of RT bubbles obtained from this spectrum is $6.4 \mu \mathrm{m}$, well under the total shell thickness (to $1 / \mathrm{e}$ of peak density) at peak velocity of $135 \mu \mathrm{m}$.

We have also simulated modulation imprint and growth with a 2-D multimode simulation. We included modes from $l=10-200$, simulated in a wedge of a sphere extending from $\theta=\pi / 2$ out to the first minimum of the $P_{10}$ Legendre polynomial, as cosine harmonics of the lowest mode. These modes are slightly different from true spherical harmonic modes, as the latter do not match a single reflection boundary in a wedge, but the difference should affect the results little. The mode amplitudes were adjusted so that the total power in all $Y_{l m}, 10 \leq l \leq 200$ was included in the reduced spectrum $(l=10,20, \ldots, 200 ; m=0)$ with the boundary equations described. We simulated imprint of static speckle with an amplitude corresponding to a $2 \mathrm{~ns}$ time-average for the beam smoothing conditions described above. Transverse modulation as a function of angle was applied in an analogous manner to the NIF imprint efficiency simulations. No surface finish modulation was included in this simulation.

The RMS modulation of the ablation front radius postprocessed from the 2-D multimode simulation is compared to two applications of the Haan quasilinear analysis in Figure 10. One Haan application uses the growth factors from the dispersion relation Eqn. 4, but applied to imprint alone. The other uses linear regime single mode growth factors from 2-D LASNEX simulations. All three cases agree to within a factor of about two. Ideally, 
the Haan application with LASNEX growth factors should agree nearly exactly with the multimode simulation as long as the perturbations are linear. However, some disagreement is expected since single mode imprint efficiencies and linear growth factors were interpolated between a subset of modes. The multimode simulation shows that the Haan theory is fairly accurate even when linear growth factors predict a high degree of saturation.

Both the Haan quasilinear model and the 2-D multimode simulation predict that ablation front modulations seeded by laser imprint do not break up the capsule shell during inward acceleration. Modulations can still feed through to the inner surface of the shell as it decelerates and generate mix into the hot spot. Our modeling to date predicts that the fairly high $l$ modes, which dominate the ablation front growth, feed through weakly on deceleration. Thus, this later phase of the implosion is less demanding upon stability than the acceleration phase for this capsule design. Those results will be presented in detail elsewhere.

\section{CONCLUSIONS}

We have simulated laser imprint for experiments carried out on the Nova laser. Simulations are in generally good agreement with experimental results for imprint of $0.35 \mu \mathrm{m}$ and $0.53 \mu \mathrm{m}$ light at $10^{13}-10^{14} \mathrm{~W} / \mathrm{cm}^{2}$ intensities, with static speckle or 1-D SSD smoothing, upon $\mathrm{CH}_{2}$ foils. We have matched the Fourier spectrum of imprint of static speckle and the improvement in RMS modulation with increasing laser bandwidth for 1-D SSD.

The time dependence of imprint has been examined for these drive conditions. Most imprint occurs in the early part of the pulse. Longer modulation wavelengths or drive with short laser wavelength cause imprint which continues later in time than shorter modulation wavelengths or longer wavelength laser drive, consistent qualitatively with the prediction of the cloudy day thermal smoothing model.

Experiments using XUV laser probes stress more elements of the modeling because of probe opacity and sample x-ray preheat issues. We have fit the RMS optical depth modu- 
lation on Si foils from static speckle but agree more poorly for SSD.

Simulations of imprint on a NIF direct drive ignition capsule have predicted the modulation spectrum resulting from imprint and RT growth at the ablation front during inward acceleration of the shell. Imprinted modulation is comparable to the surface finish expected from target fabrication, about $10 \mathrm{~nm}$ RMS. Linear growth factors at modes $l \sim 100-200$ reach into the thousands, but nonlinear saturation mitigates the effects. Shells are predicted to remain intact, which gives us a basis for believing that ignition can be achieved.

\section{ACKNOWLEDGMENTS}

We thank the many contributors to the experiments on the Nova and Vulcan lasers, who are credited in the references reporting on the individual experiments. ${ }^{13,6,7,10}$ This work was

performed under the auspices of the U.S. Department of Energy by the Lawrence Livermore National Laboratory under contract number W-7405-ENG-48. 


\section{REFERENCES}

${ }^{1}$ Y. Kato, K. Mima, N. Miyanaga, S. Aringa, Y. Kitagawa, M. Nakatsuka, and C. Yamanaka, Phys. Rev. Lett. 53, 1057 (1984).

${ }^{2}$ S. Skupsky, R. W. Short, T. Kessler, R. S. Craxton, S. Letring, and J. M. Soures, J. Appl. Phys. 66, 3456 (1989).

${ }^{3}$ R. H. Lehmberg and S. P. Obenschain, Optics Comm. 46, 27 (1983).

${ }^{4}$ S. E. Bodner, J. Fusion Energy 1, 221 (1981).

${ }^{5}$ M. Desselberger, T. Afshar-rad, F. Khattak, S. Viana, and O. Willi, Phys. Rev. Letters 68, $1539(1992)$.

${ }^{6}$ S. G. Glendinning, S. N. Dixit, B. A. Hammel, D. H. Kalantar, M. H. Key, J. D. Kilkenny, J. P. Knauer, D. M. Pennington, B. A. Remington, R. J. Wallace, and S. V. Weber, Phys. Rev. E 54, 4473 (1996).

${ }^{7}$ D. H. Kalantar, M. H. Key, L. B. DaSilva, S. G. Glendinning, J. P. Knauer, B. A. Remington, F. Weber, and S. V. Weber, Phys. Rev. Lett. 76, 3574 (1996).

${ }^{8}$ R. J. Taylor, J. P. Dahlburg, A. Iwase, J. H. Gardner, D. E. Fyfe, and O. Willi, Phys. Rev. Lett. 76, 164, (1996).

${ }^{9}$ T. R. Boehley, D. D. Meyerhofer, J. P. Knauer, D. K. Bradley, R. L. Keck, J. A. Delettrez, V. A. Smalyuk, J. M. Soures, and C. P. Verdon, Bull. Am. Phys. Soc., 41 (1996).

${ }^{10}$ D. H. Kalantar, M. H. Key, L. B. DaSilva, S. G. Glendinning, B. A. Remington, J. E. Rothenberg, F. Weber, S. V. Weber, E. Wolfrum, N. S. Kim, D. Neely, J. Zhang, J. S. Wark, A. Demir, J. Lin, R. Smith, G. J. Tallents, C. L. S. Lewis, A. MacPhee, J. Warwich, and J. P. Knauer, "Measurements of direct drive laser imprint in thin toils by XUV radiography using an x-ray laser backlighter", submitted to Phys. Plasmas.

${ }^{11}$ C. Pawley, "Measurements on Nike of Rayleigh-Taylor growth of target and laser imprinted 
perturbations", submitted to Phys. Plasmas.

${ }^{12}$ H. Azechi, "Direct drive hydrodynamic instability experiments", submitted to Phys. Plasmas.

${ }^{13}$ S. G. Glendinning, S. N. Dixit, B. A. Hammel, D. H. Kalantar, M. H. Key, J. D. Kilkenny, J. P. Knauer, D. M. Pennington, B. A. Remington, R. J. Wallace, S. V. Weber, “A comparison of drive-seeded modulations in planar foils for 0.35 and $0.53 \mu \mathrm{m}$ laser drive", to be submitted to Phys. Plasmas.

${ }^{14}$ M. H. Emery, J. H. Gardner, R. H. Lehmberg, and S. P. Obenschain, Phys. Fluids B 3, $2640(1991)$.

${ }^{15}$ G. B. Zimmerman and W. L. Kruer, Comment Plasma Phys. Controlled Fusion, 2, 51 $(1975)$.

${ }^{16}$ S. G. Glendinning, S. V. Weber, P. Bell, L. B. DaSilva, S. N. Dixit, M. A. Henesian, D. R. Kania, M. H. Key, J. D. Kilkenny, H. T. Powell, R. J. Wallace, P. J. Wegner, J. P. Knauer, C. P. Verdon, Phys. Rev. Lett. 69, 120, (1992).

${ }^{17}$ S. G. Glendinning, S. N. Dixit, B. A. Hammel, D. H. Kalantar, M. H. Key, J. D. Kilkenny, J. P. Knauer, D. M. Pennington, B. A. Remington, R. J. Wallace, and S. V. Weber, "Measurements of linear regime Rayleigh-Taylor growth rates in laser-driven planar targets", submitted to Phys. Rev. E.

${ }^{18}$ C. A. Iglesias, F. J. Rogers, and B. G. Wilson, Astroph. J. 397, 717 (1992); ibid. 360, 221 (1990); ibid. 322, L45 (1987); F. J. Rogers and C. A. Iglesias, Astroph. J. Suppl. 79, 507 (1992). (1992).

${ }^{19}$ G. B. Zimmerman and R. M. More, J. Quant. Spectrosc. Radiat. Transfer 23, 517 (1980); R. M. More, ibid. 27, 345 (1982).

${ }^{20}$ S. W. Haan, S. M. Pollaine, J. D. Lindl, L. J. Suter, R. L. Berger, L. V. Powers, W. E. 
Alley, P. A. Amendt, J. A. Futterman, W. K. Levedahl, M. D. Rosen, D. P. Rowley, R. A. Sacks, A. I. Shestakov, G. L. Strobel, A. Tabak, S. V. Weber, G. B. Zimmerman, W. J. Krauser, D. C. Wilson, S. V. Coggeshall, D. B. Harris, N. M. Hoffman, and B. H. Wilde, Phys. Plasmas 2, 2480 (1995).

${ }^{21}$ C. P. Verdon, Bull. Am. Phys. Soc. 38, 2010 (1993).

${ }^{22}$ J. E. Rothenberg, Proc. Soc. Photo-Opt. Instrum. Eng. 2633, 634 (1995).

${ }^{23}$ H. Takabe, L. Montierth, and R. L. Morse, Phys. Fluids 26, 2299 (1983); H. Takabe, K. Mima, L. Montierth, and R. L. Morse, ibid. 28, 3676 (1985).

${ }^{24}$ J. Lindl, Phys. Plasmas 2, 3933 (1995).

${ }^{25}$ S. W. Haan, Phys. Rev. A 39, 5812 (1989).

${ }^{26}$ M. J. Dunning and S. W. Haan, Phys. Plasmas 2, 1669, (1995).

${ }^{27}$ S. W. Haan (private communication, 1996). 


\section{FIGURES}

FIG. 1. Measured and simulated imprint efficiency amplitude spectrum( $\mu \mathrm{m})$ vs. mode number for static speckle, $0.35 \mu \mathrm{m}$ or $0.53 \mu \mathrm{m}$ illumination.

FIG. 2. Modulation in column density vs. time for modulation wavelength $100 \mu \mathrm{m}$, illumination wavelength $0.35 \mu \mathrm{m}$. In the uppermost curve the modulation is applied at all times, whereas the illumination becomes uniform at $1 \mathrm{~ns}, 0.5 \mathrm{~ns}, 0.25 \mathrm{~ns}$, and $0.1 \mathrm{~ns}$ for the other cases.

FIG. 3. Imprint efficiency vs. imprinting time for illumination wavelengths of $0.35 \mu \mathrm{m}$ and 0.53 $\mu \mathrm{m}$, modulation wavelengths of $30 \mu \mathrm{m}$ and $100 \mu \mathrm{m}$.

FIG. 4. Equivalent surface finish RMS vs. laser bandwidth for $20 \mu \mathrm{m}$ thick $\mathrm{CH}_{2}$ foils, 1-D SSD, $0.53 \mu \mathrm{m}$ laser drive at up to $10^{14} \mathrm{~W} / \mathrm{cm}^{2}$.

FIG. 5. Measured and simulated RMS optical depth modulation in Si foils vs. SSD bandwidth. Measured optical intensity modulation is also shown, as well as the expected value at zero bandwidth of unity.

FIG. 6. NIF direct drive ignition capsule design and pulse shape. The capsule is illuminated with 1.5 MJ of $0.35 \mu \mathrm{m}$ laser light and gives a calculated yield of 17 MJ.

FIG. 7. Imprint efficiency vs. spherical harmonic mode number $l$ from planar simulations of imprint upon the NIF capsule shell.

FIG. 8. Equivalent surface finish mode spectrum, fabrication surface finish spectrum, and RMS sum for the NIF direct drive capsule design.

FIG. 9. Spectrum of perturbation amplitude at the ablation front at peak shell velocity for the sum of imprint and surface finish. Shown are amplitudes deduced from linear growth factors (solid), amplitude of saturation onset (dotted), and amplitudes corrected for saturation (dashed). 
FIG. 10. Modulation RMS amplitude vs. time at the ablation front postprocessed from a LASNEX 2-D multimode simulation (2-D MM), and from application of the $\mathrm{Haan}^{25}$ model using linear growth factors from either Eqn. 4 (analytic) or from LASNEX single mode simulations. An estimate of the shell thickness is also shown. 


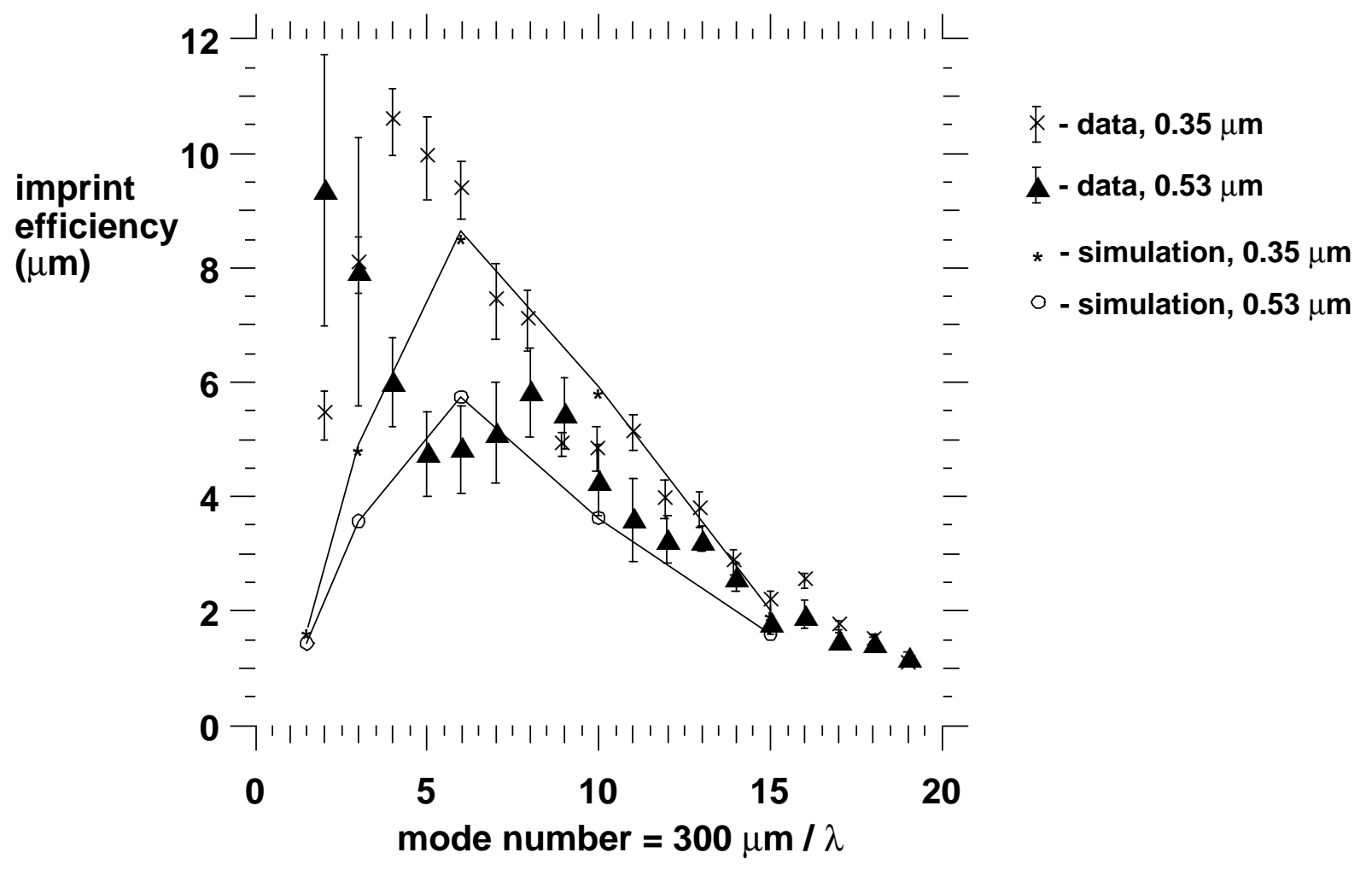

Figure 1 


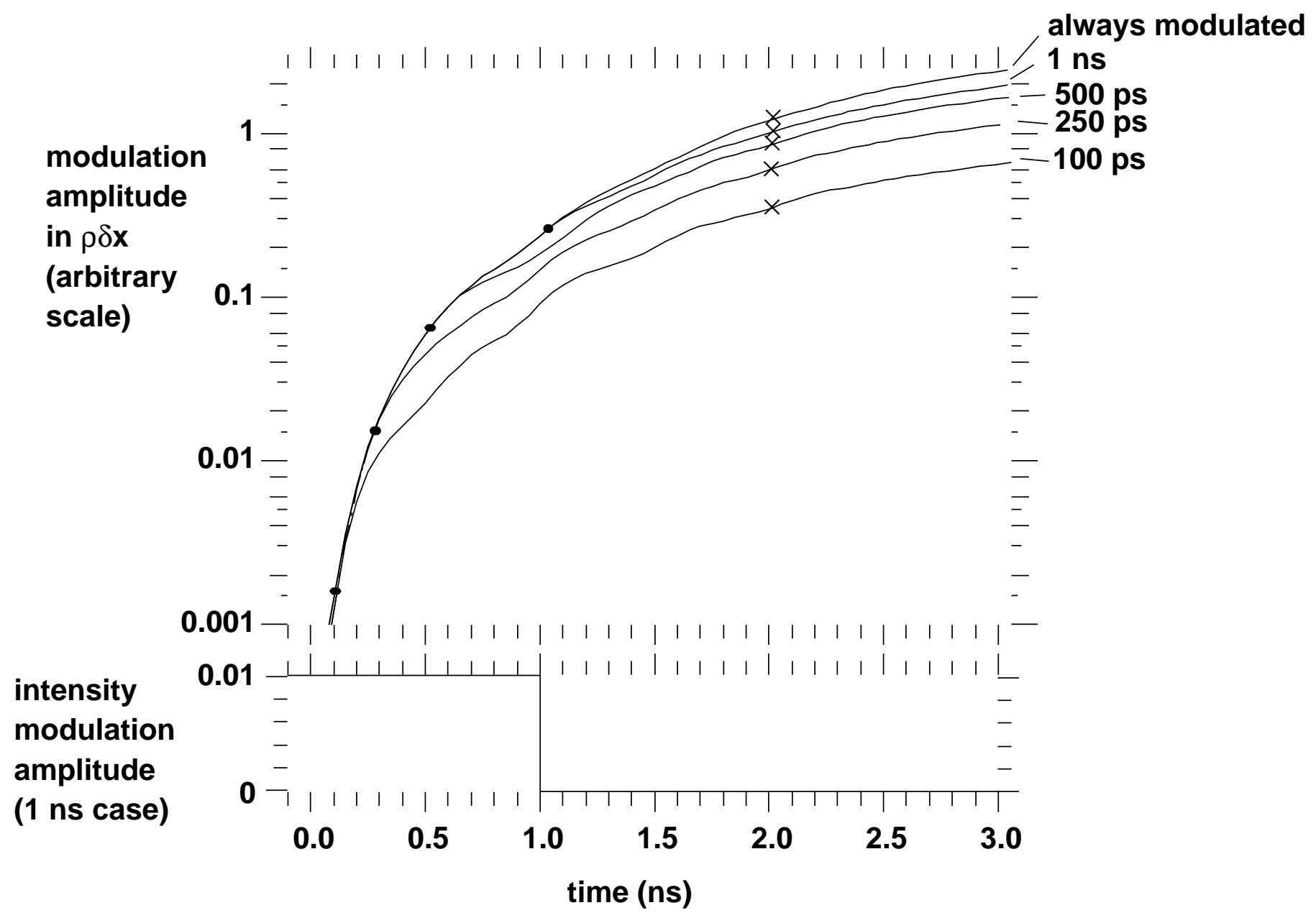

Figure 2 


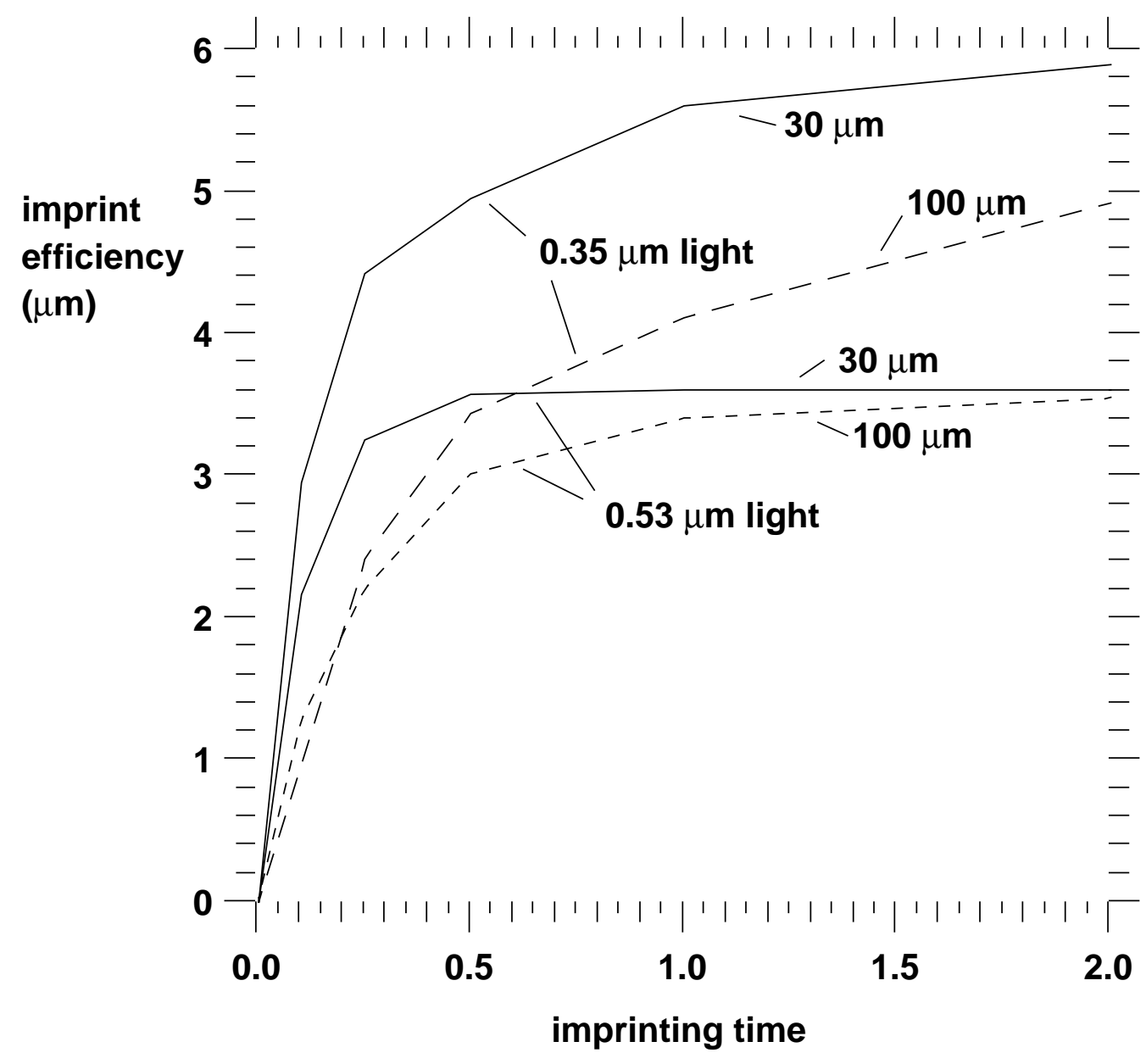

Figure 3 


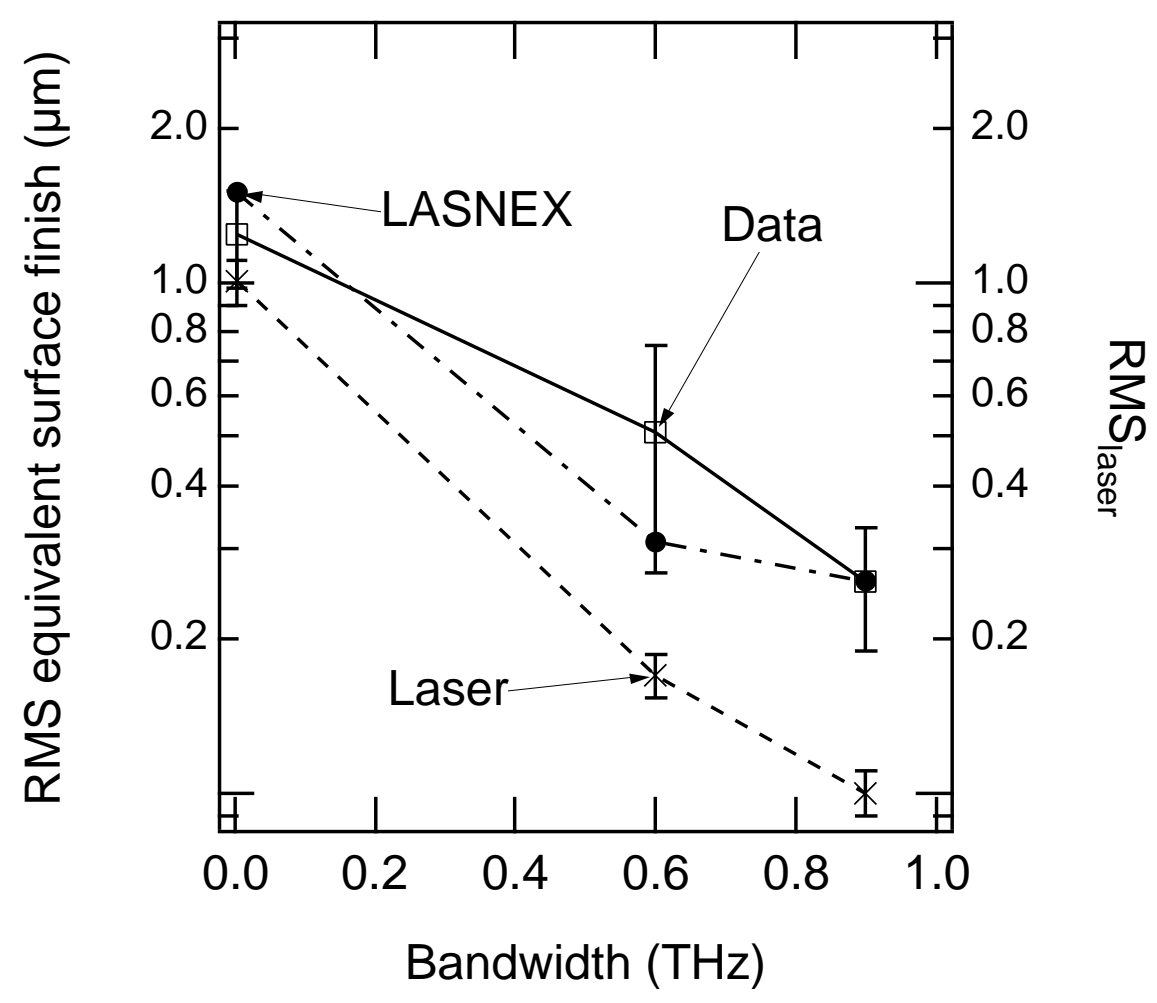




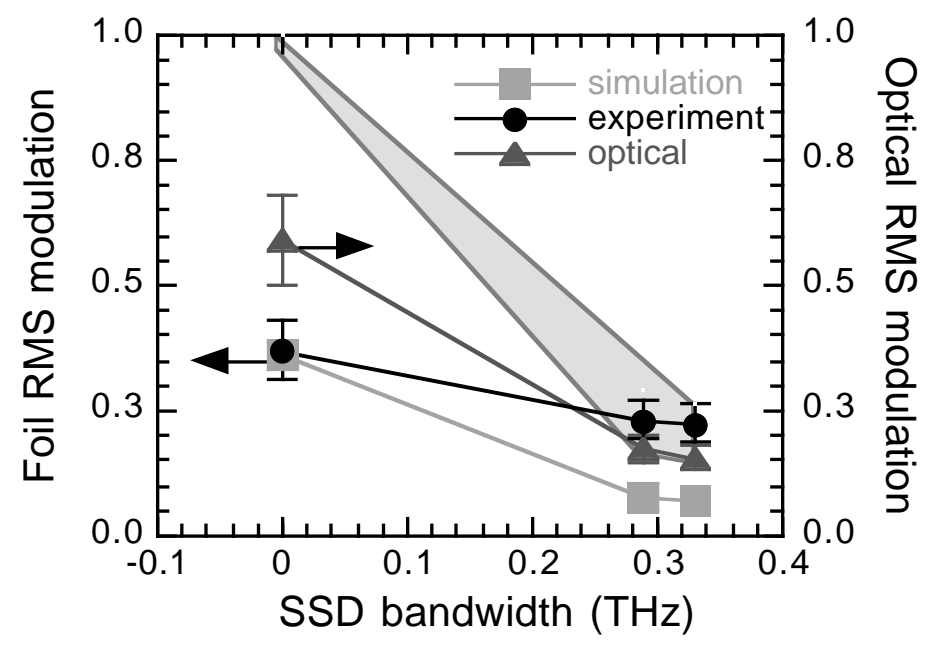

Figure 5 
Pulse shape, $0.35 \mu \mathrm{m}$ laser

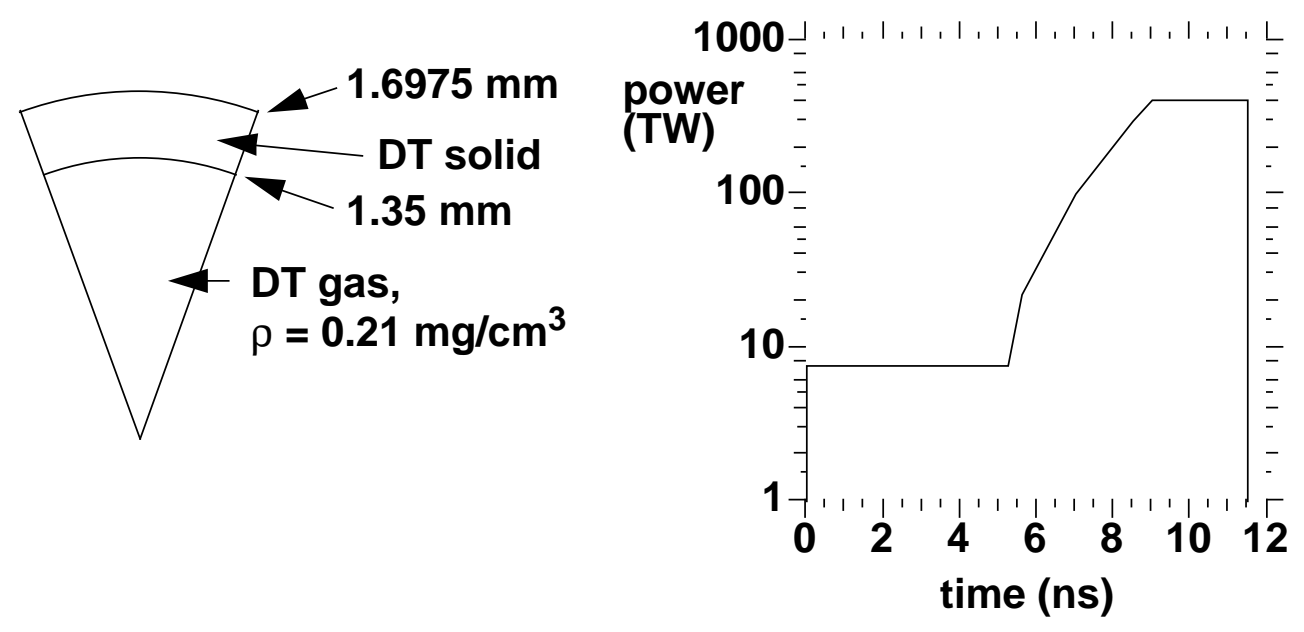

Figure 6 


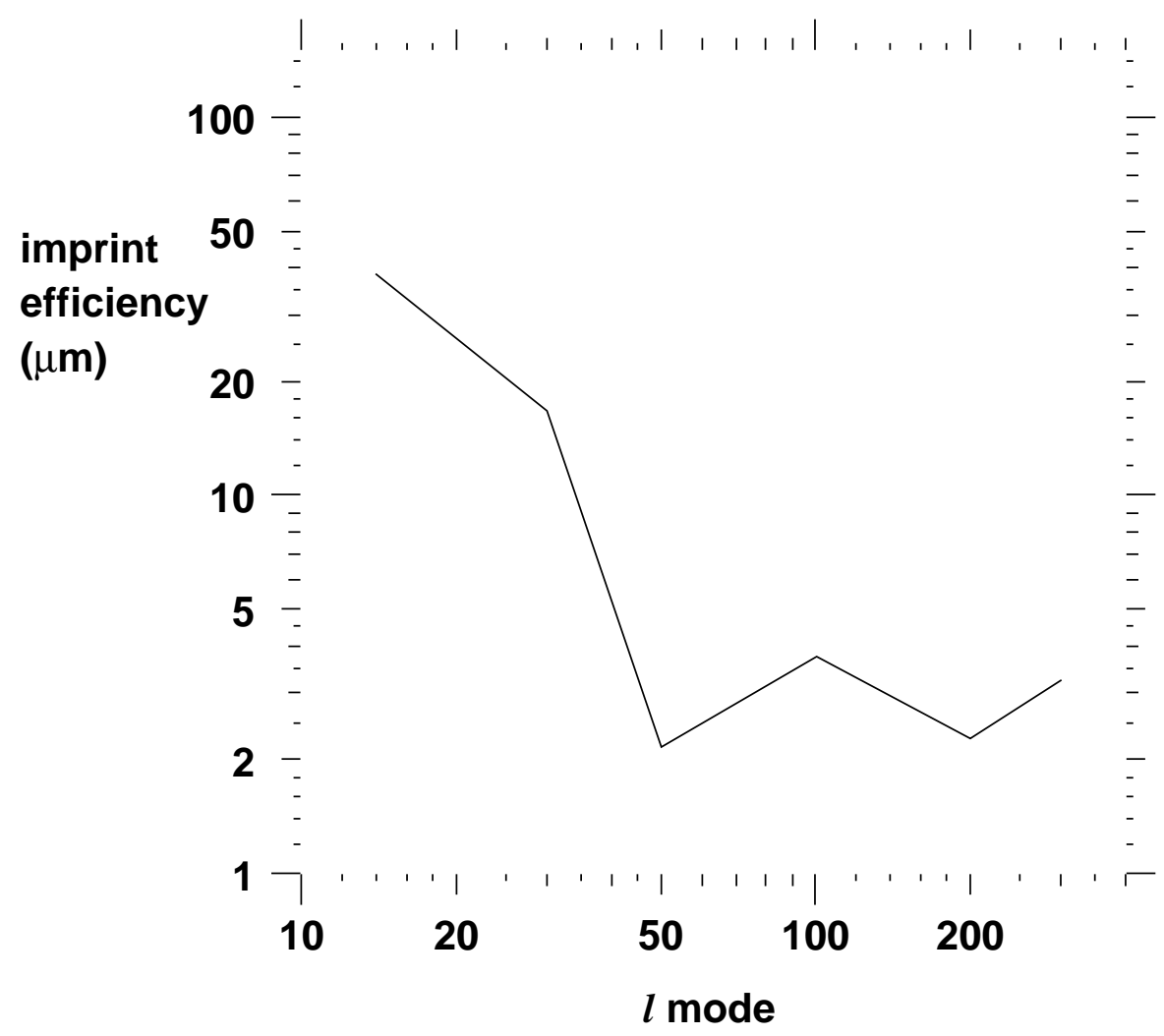

Figure 7 


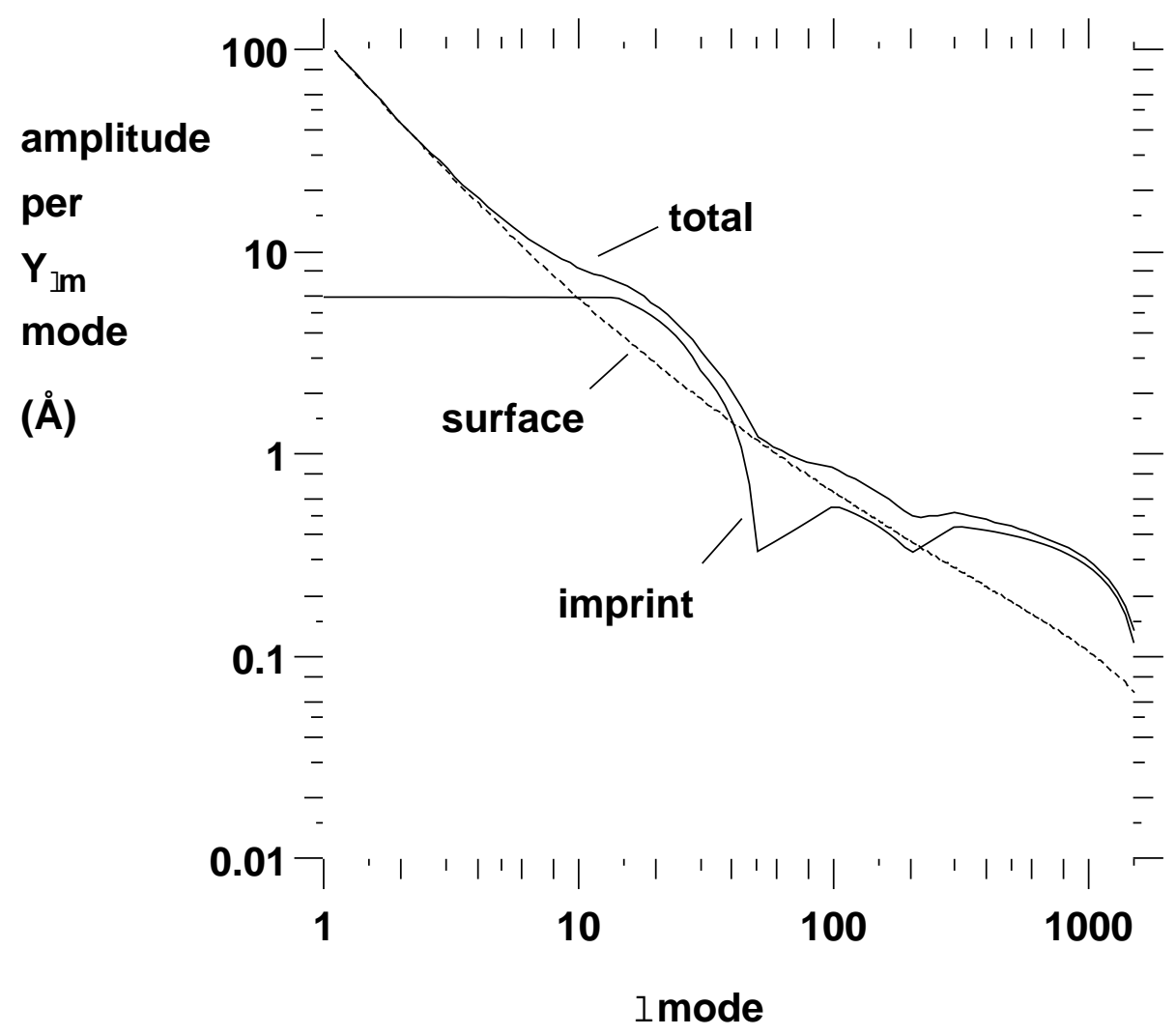

Figure 8 


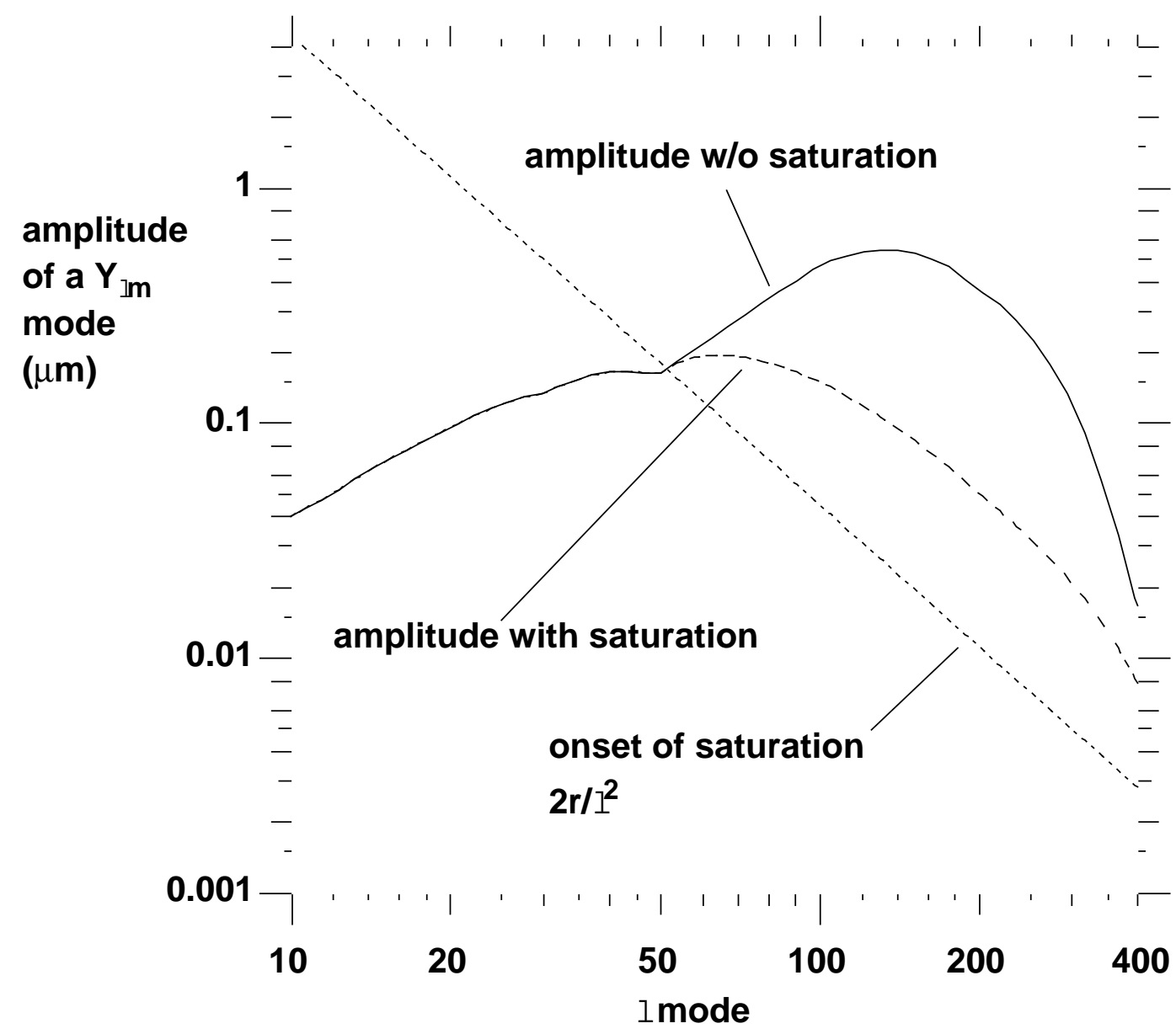

Figure 9 


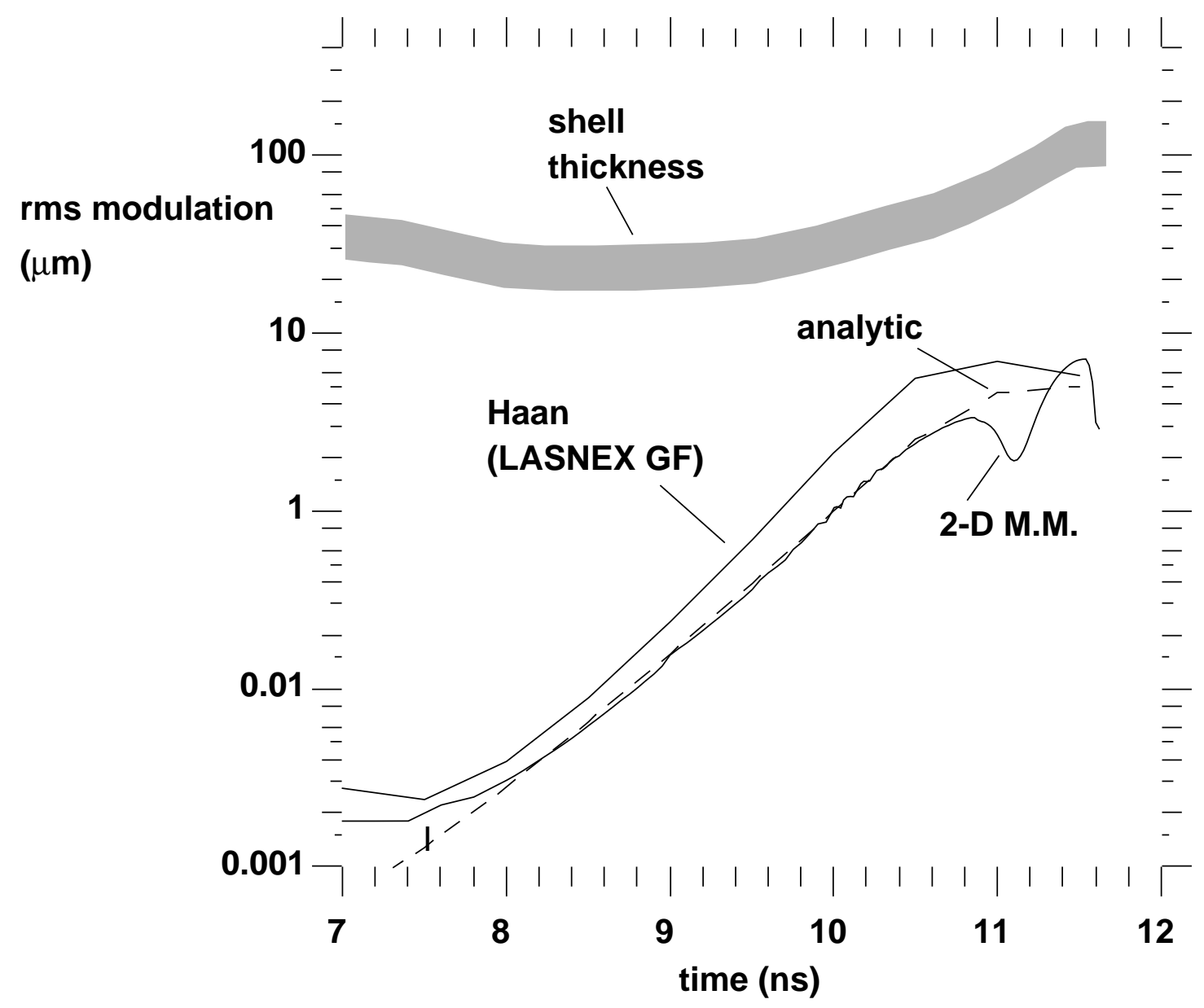

Figure 10 


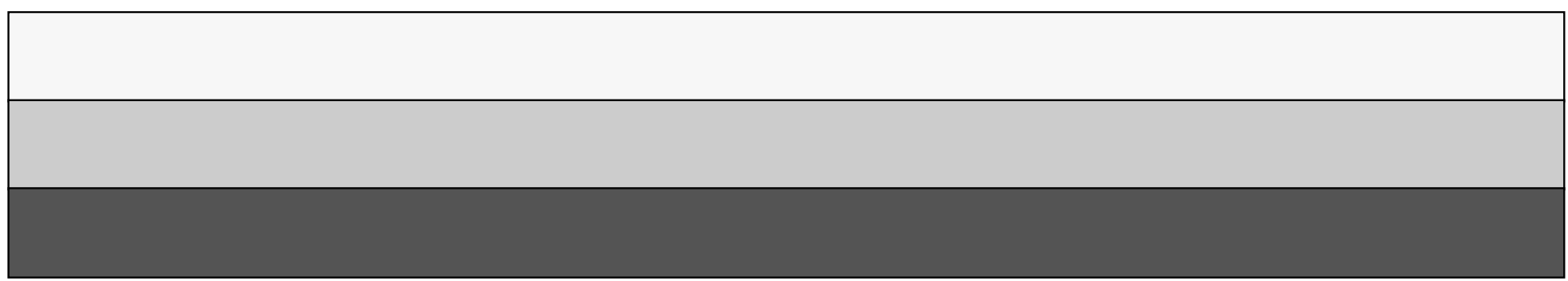

